\section{Obstetric anaesthesia and MoyaMoya disease}

To the Editor:

We read with interest the letter from Drs. Venkatesh and Taggart ${ }^{1}$ regarding the anaesthetic management of a patient with MoyaMoya disease during Caesarean section. These authors described successful general anaesthesia for this patient and claim it to be the ideal anaesthetic technique. However, there was no discussion of the benefits of regional anaesthesia in this patient. We recently used epidural anaesthesia for a term parturient with MoyaMoya disease undergoing Caesarean section. The outcome was successful and we would like to defend our rationale for using a regional technique in this patient.

The patient with MoyaMoya disease has a compromised cerebral circulation. ${ }^{2}$ There may be an associated intracranial aneurysm, the incidence of which has been estimated to be $14 \%$ in adults. ${ }^{3}$ Identifiable factors precipitating neurological deficit during anaesthesia in these patients include hyperventilation resulting in hypocarbia, ${ }^{4}$ severe hypotension, and subarachnoid haemorrhage due to sudden fluctuation in systemic arterial pressure. ${ }^{5}$

In the obstetric population, central neural block is preferred over general anaesthesia for elective Caesarean section. The risk of maternal aspiration and neonatal drug depression is thereby reduced, and factors that increase cerebral metabolic oxygen consumption, such as the stress response to laryngoscopy and tracheal intubation, are avoided during regional anaesthesia. The hypertensive response to intubation may be detrimental particularly in such cases as MoyaMoya disease with associated intracranial aneurysms, and may increase the risk of intracerebral haemorrhage. Also, during regional anaesthesia, the anaesthetist remains in communication with the patient, and can monitor cerebral function by observing the level of consciousness and the appearance of any abnormal neurological signs.

However, MoyaMoya vessels and other normal collateral vessels in the ischaemic hemisphere are maximally dilated. We agree with the authors that, in these patients, it is especially important to avoid hypotension in order to maintain cerebral blood flow and to prevent further ischaemia. Although epidural anaesthesia may be associated with hypotension, incremental dosing through a catheter and appropriate fluid preloading minimizes this risk. We used epidural anaesthesia in this way and successfully avoided extensive sympathetic blockade and hypotension. Uterine displacement was maintained throughout the procedure. We also catheterized a radial artery for continuous monitoring for hypotension and regular measurement of arterial $\mathrm{CO}_{2}$ tension. The patient re- mained stable during the operation and made an uneventful recovery. Spinal anaesthesia is best avoided in these patients because of a greater risk of hypotension.

Hypocarbia during controlled ventilation in a patient with MoyaMoya disease has been associated with signs of cerebral ischaemic such as seizures, therefore avoidance of hypocarbia and cerebral vasoconstriction is recommended. ${ }^{4}$ In our patient, normocarbia was maintained during the epidural anaesthetic.

We believe that, in the absence of a contraindication, epidural anaesthesia is a better option for Caesarean section in a MoyaMoya patient.

Shiv K. Sharma MB BS MD FRCA

Donald H. Wallace MD

J. Elaine Sidaiwi MD

David R. Gambling MB BS FRCPC

Department of Anesthesiology and Pain Management

University of Texas Southwestern Medical Center

5323 Harry Hines Boulevard

Dallas, TX 75235-9068

\section{REFERENCES}

1 Venkatesh B, Taggart PCM. Anaesthetic management of a patient with moyamoya disease for Caesarean section. Can J Anaesth 1994; 41: 79-80.

2 Ogawa A, Yoshimoto T, Suzuki J, Sakurai Y. Cerebral blood flow in moyamoya disease. Part 1 . Correlation with age and regional distribution. Acta Neurochir (Wien) 1990; 105: 30-4.

3 Konishi Y, Kadowaki C, Hara M, Takeuchi $K$. Aneurysms associated with moyamoya disease. Neurosurgery 1985; 16: 484-91.

4 Bingham RM, Wilkinson DJ. Anaesthetic management of moya-moya disease. Anaesthesia 1985; 40: 1198-202.

5 Hashimoto K, Fujii K, Nishimura K, Kibe M, Kishikawa $T$. Occlusive cerebrovascular disease with moyamoya vessels and intracranial haemorrhage during pregnancy - case report and review of the literature. Neurologia MedicoChirurgica 1988; 28: 588-93.

\section{$R E P L Y$}

We would like to thank Drs. Sharma et al. for their comments on our letter.' We described an anaesthetic technique which resulted in a satisfactory outcome, but did not claim it to be the ideal technique. We discussed the potential hazards of general anaesthesia in this group of patients in our report and took appropriate precautions. We are delighted that the case report by Drs. Sharma et al. provides valuable evidence that regional blocks may also be a safe and suitable method of anaesthesia for Caesarean section in patients with MoyaMoya disease. As this is an uncommon disease, the continued accumulation of evidence for the suitability of various anaesthetic techniques, such as the above, is essential for individualizing anaesthetic management in this group of patients. We would like to emphasize, however, that it is the safe and careful con- 